\title{
Direct observation of fine-scale energy banding in echo-enabled harmonic generation
}

\author{
E. Hemsing, D. Xiang, M. Dunning, S. Weathersby, C. Hast, and T. Raubenheimer \\ SLAC National Accelerator Laboratory, Menlo Park, California 94025, USA
}

(Received 5 July 2013; published 30 January 2014)

\begin{abstract}
We describe the experimental observation of highly nonlinear energy striations generated by two lasers in a relativistic electron beam in an echo-enabled harmonic generation (EEHG) setup. The rich energy banding structure results from strong dispersion of the sinusoidally modulated beam, and measurements of the banding spectrum enable benchmarking, optimization, and characterization of the concomitant EEHG process. Results are found to be in good agreement with theory, and suggest that the presented technique can facilitate the practical implementation of EEHG to generate intense, fully coherent light in future advanced accelerator-based light sources.
\end{abstract}

DOI: 10.1103/PhysRevSTAB.17.010703

PACS numbers: 41.60.Cr, 42.65.Ky

\section{INTRODUCTION}

Echo-enabled harmonic generation (EEHG) is a proposed method for particle accelerator-based light sources to produce intense, fully coherent radiation at $x$-ray wavelengths $[1,2]$. In modern high-gain $\mathrm{x}$-ray free electron lasers (FELs), the radiation typically starts from shot noise in the electron beam (e-beam), which results in limited temporal coherence and large statistical fluctuations in the output light. To overcome these limitations, EEHG and other external seeding schemes [3,4] use laser modulators and dispersive sections to produce a high-harmonic current modulation in the e-beam, which then acts as the fully coherent seed for the exponentially amplified radiation. The EEHG proposal has drawn international interest [5-12], and while the preliminary concept has been demonstrated at the third [13,14] and seventh [15] harmonics of IR lasers, it is still an open question whether EEHG can be pushed to much higher harmonics and shorter wavelengths. A particularly challenging concern is the preservation of the highly nonlinear energy striations generated in the e-beam longitudinal phase space, which may be degraded by intrabeam scattering (IBS), incoherent synchrotron radiation (ISR), and other transport effects [2,16,17]. Accordingly, direct experimental access to these structures would be a useful tool to directly characterize both the EEHG process and unwanted effects, as well as to optimize its implementation in practice.

In this paper, we demonstrate a new method aimed to serve these purposes through the direct measurement of the fine-scale structures generated in the e-beam energy domain at an intermediate stage of the EEHG

Published by the American Physical Society under the terms of the Creative Commons Attribution 3.0 License. Further distribution of this work must maintain attribution to the author(s) and the published article's title, journal citation, and DOI. up-conversion process. This technique, which we refer to as EEHG in the energy space, or EEHG-E, enables direct, broadband diagnosis of the multiple energy striations generated during EEHG that encode the harmonic frequency content. It also provides a direct way to measure the laser modulation and dispersion values that are key to EEHG optimization. With sufficient energy resolution, this technique could also be used to experimentally quantify deleterious effects such as IBS and ISR that set fundamental limits on the achievable harmonics.

The schematic layout of the setup and evolution of the ebeam phase space is shown in Fig. 1. In EEHG, a relativistic e-beam propagating through a magnetic undulator is sinusoidally modulated in energy by a copropagating laser with frequency $k_{1}=2 \pi / \lambda_{1}$ [Fig. 1(A)]. The beam is then strongly overdispersed in a chicane with momentum compaction factor $R_{56}$ [Fig. 1(B)]. Electrons with higher energy slide forward with respect to electrons with lower energy, which creates the strongly sheared longitudinal phase space distribution with many correlated energy bands. In EEHG, the beam is then again modulated by a second laser at frequency $k_{2}=2 \pi / \lambda_{2}$, and then dispersed in a weaker chicane [Fig. 1(C)] to convert the fine-scale energy striations into a high-harmonic density modulation [Fig. 1(D)] at the frequency $a k_{1}$, where $a \gg 1$ is the harmonic factor. The resulting harmonic bunching spectrum is shown in Fig. 1(E). The microbunched e-beam can then emit intense coherent light at the harmonic wavelengths in an FEL undulator.

In our EEHG-E setup however, we impart a smaller modulation with the second laser to simply flatten the energy bands [Fig. 1(F)]. This removes the longitudinal correlation in specific regions of the beam so that the bands are observable in the projected e-beam energy space at a magnetic bend spectrometer screen far downstream. The calculated projected energy distribution is shown in Fig. 1(G). This procedure enables direct measurement of 

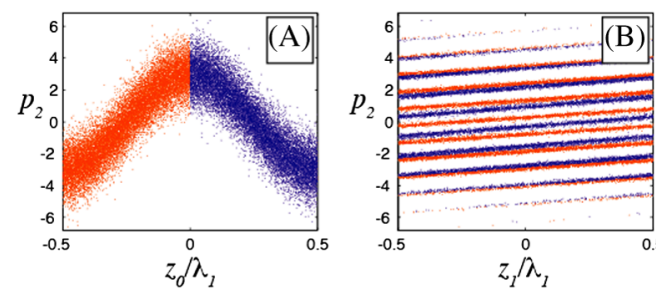

$z_{1} / \lambda_{1}$

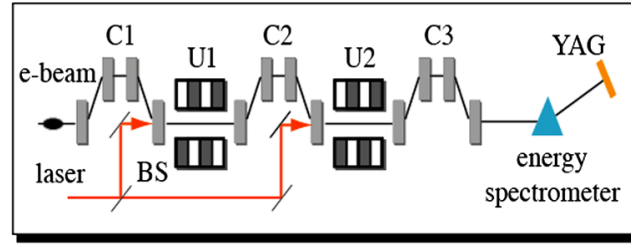

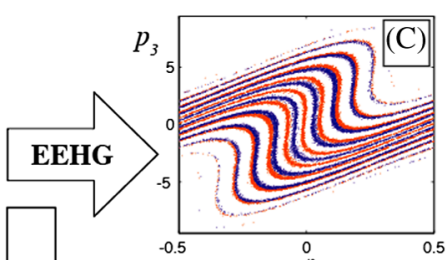

$z_{2} / \lambda_{1}$

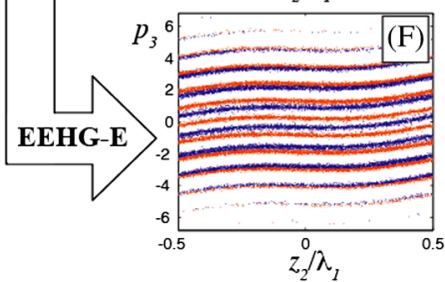

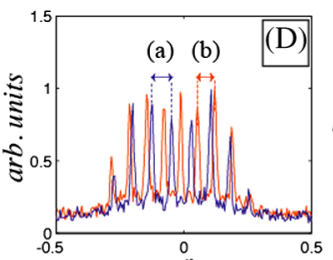

$z_{2} / \lambda$

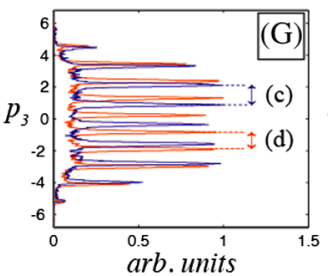

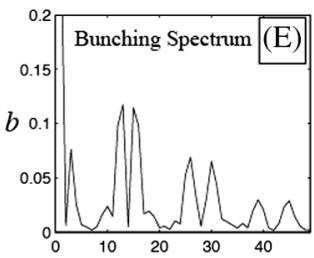

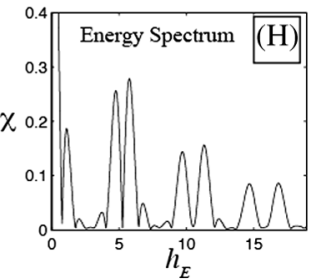

FIG. 1 (color online). Lower left: Diagram of the EEHG-E experiment at the Next Linear Collider Test Accelerator (NLCTA). The sinusoidal energy modulation (A) generated in U1 is strongly dispersed (B) as the beam travels through C2. In U2, a second modulation is used to either rotate the energy bands upright after C3 (C) to generate harmonic density bunching (D) for EEHG, or to flatten the energy bands $(\mathrm{F})$ to generate observable peaks in the projected energy $(\mathrm{G})$ at the YAG screen in EEHG-E. Blue and red regions of different initial chirp lead to differently spaced peaks in the energy projection [(c) and (d), respectively] or density projection [(a) and (b)]. The harmonic frequency content of the EEHG density bunching (E) and EEHG-E energy banding (H) show similar structure.

the individual energy bands generated in the first modulator/chicane section, which we show are closely connected with the harmonic bunching structure in EEHG. The underlying physical correspondence between the EEHG bunching and the EEHG-E projected energy distributions is highlighted by their similar spectra [Figs. 1(E) and 1(H)] which contain multiple pairs of peaks with comparable structure. One advantage of this arrangement is that, in EEHG, experimental observation of the final bunching spectrum is indirect and often difficult, as one can only infer the bunching spectrum from the radiation emitted by the beam, which is typically over only a narrow frequency range at suboptical wavelengths. In contrast, the corresponding energy banding spectrum can be directly measured through EEHG-E over a large range of harmonics simultaneously, thus making it a broadband experimental probe of the EEHG process.

\section{ANALYTIC DESCRIPTION}

Our experiment is guided by mathematical analysis which closely follows that of EEHG [1,2]. We consider an electron beam with energy $E_{0}$ and energy spread $\sigma_{E}$. The initially uncorrelated distribution is described by a product of Gaussians in energy and longitudinal position as $f\left(p_{0}, z_{0}\right)=\left(2 \pi \sigma_{z}\right)^{-1} e^{-p_{0}^{2} / 2} e^{-z_{0}^{2} / 2 \sigma_{z}^{2}}$, where $p_{0}=$ $\left(E-E_{0}\right) / \sigma_{E}$ is the initial relative energy deviation of an electron, $z_{0}$ is its position in the beam, and $\sigma_{z}$ is the rms bunch length. We also include the effects of the rf accelerating fields on the initial beam distribution via the relation $p_{1}=p_{0}-h_{1} k_{1} z_{0}-h_{2} k_{1}^{2} z_{0}^{2}$, where $h_{1}=$ $2 \pi q V \sin \left(\varphi_{\mathrm{rf}}\right) / \sigma_{E} \lambda_{\mathrm{rf}} k_{1}$ is the scaled linear bunch chirp (i.e., the linear correlation between energy $E$ and longitudinal position $z_{0}$ over the entire bunch) from an rf linac with accelerating energy $q V$, wavelength $\lambda_{\text {rf }}$, and off-crest phase $\varphi_{\mathrm{rf}}$. Similarly, the quadratic component responsible for phase space curvature over the whole bunch is $h_{2}=(2 \pi)^{2} q V \cos \left(\varphi_{\mathrm{rf}}\right) / 2 \sigma_{E} \lambda_{\mathrm{rf}}^{2} k_{1}^{2} q$. The first laser then modulates the energy of the e-beam by $p_{2}=p_{1}+A_{1} \sin \left(k_{1} z_{0}\right)$, where $A_{1}=\Delta E_{1} / \sigma_{E}$ is the scaled modulation amplitude. The beam is then longitudinally dispersed in a chicane, and the longitudinal coordinate is transformed according to $z_{1}=z_{0}+B_{1} p_{2} / k_{1}$, where $B_{1}=$ $k_{1} R_{56} \sigma_{E} / E_{0}$ is the scaled momentum compaction. As with EEHG, a second laser modulates the beam as $p_{3}=p_{2}+A_{2} \sin \left(k_{2} z_{1}+\phi\right)$, where $A_{2}=\Delta E_{2} / \sigma_{E}$ and $\phi$ is the phase with respect to the first laser. The transformation performed on the longitudinal phase space by the second chicane $\left(B_{2}=k_{1} R_{56}^{(2)} \sigma_{E} / E_{0}\right)$ in EEHG is ignored here, as it does not modify the projected energy distribution. Analogous to the familiar bunching factor, $b$, that describes the EEHG harmonic density bunching amplitude, here we define an energy banding spectrum,

$$
\chi(\mu)=\left|\int e^{i \mu p_{3}\left(p_{0}, z_{0}\right)} f\left(p_{0}, z_{0}\right) d p_{0} d z_{0}\right|
$$

which quantifies the banding of the e-beam energy as a function of the scaled variable $\mu=\kappa \sigma_{E}$, where $\kappa=2 \pi / \delta E$ is referred to as the frequency of the banding in the energy space, with bands separated by $\delta E$.

Evaluation of Eq. (1) for arbitrary values of dispersion and amplitude modulation is given in Eq. (A1) in the Appendix. For the purposes of this analysis, we concentrate only on parameter values relevant to EEHG and our setup; namely, that the bunch length is long compared to the laser wavelengths $\left(\sigma_{z} k_{1,2} \gg 1\right)$, that $A_{2} \lesssim A_{1}$, that the shearing in the phase space is large $\left(A_{1} B_{1} \gg 1\right)$ so that the relative phase $\phi$ between lasers is inconsequential, and that the 
linear and quadratic rf contributions $h_{1}$ and $h_{2}$ are negligible. Further, in our experiment the lasers have the same wavelength, $k_{1}=k_{2}$. Under these conditions the energy banding spectrum has a similar form to the EEHG bunching factor $[1,2]$ and is given by

$$
\chi(\mu)=\left|\sum_{n} \operatorname{Exp}\left[-\frac{\left(\mu-n B_{1}\right)^{2}}{2}\right] J_{n}\left(\mu A_{1}-n B_{1} A_{1}\right) J_{-n}\left(\mu A_{2}\right)\right| .
$$

Analysis shows that $\chi(\mu)$ in Eq. (2) has multiple peaks near the scaled frequencies $\mu_{n}=n B_{1}$ corresponding to energy separations of $\delta E_{n}=2 \pi / \kappa_{n}=\lambda_{1} E_{0} / n R_{56}$, which decrease as the first chicane strength is increased. At these frequencies, Eq. (2) indicates that the first Bessel function vanishes exactly for $n \neq 0$, so $\mu_{n}$ identifies zeros in the EEHG-E frequency spectrum that are surrounded by multiple nearby peaks, as seen in Fig. 1(H).

The origin of these multiple sets of peaks in the banding spectrum is relevant for benchmarking the subtle physics of EEHG where they also appear in the bunching spectrum, so it is instructive to examine how they occur. The physical picture is illustrated in Fig. 1(A). The first laser modulation generates regions of both positive (red) and negative (blue) local chirp on the scale of the laser wavelength (i.e., over much smaller scales than the global chirp due to rf) that overlap each other longitudinally after strong dispersion [Fig. 1(B)]. The relative energy separation between neighboring bands with the same initial positive or negative local chirp is $\Delta p_{ \pm} \simeq\left|2 \pi A_{1} /\left(1 \pm A_{1} B_{1}\right)\right|$. After the second smaller modulation $A_{2}$ in EEHG-E [Fig. 1(F)] that flattens out the energy bands, the different projected energy spacings [marked as (c) and (d) in Fig. 1(G)], produce the pair of distinct banding frequencies in the energy spectrum near $\kappa_{1}$ and the $n$th harmonics $\kappa_{n}$ [Fig. 1(H)]. In total there are up to $2 A_{1} B_{1} / \pi$ energy bands across the distribution, which we quantify by defining a harmonic number in the energy space, $h_{E}=2 \Delta E_{1} / \delta E$. This describes the number of energy oscillations in the distribution, which is found from the harmonic peaks in the banding spectrum via $h_{E}=\kappa_{n} \Delta E_{1} / \pi=n A_{1} B_{1} / \pi$. In EEHG, the energy bands are rotated upright by the action of the larger second laser modulation and subsequent chicane (with $A_{2} \simeq 1 / B_{2}$ ) to generate density bunching. Analogously, two distinct longitudinal spacings $\Delta z \simeq$ $\lambda_{1} / a_{ \pm}$between density spikes emerge [(a) and (b) in Fig. 1(D)], which produce neighboring peaks in the harmonic microbunching spectrum at $a_{ \pm} \simeq\left|\left(1 \pm A_{1} B_{1}\right)\right|$ $A_{1} B_{2} \mid$ [Fig. 1(H)]. EEHG is typically optimized to select only one of these peaks to radiate coherently [18] . In contrast, from the projected energy spectrum we see that broadband features of the EEHG process can be examined explicitly. Further, the banding harmonic number is related to the EEHG harmonic number as $h_{E} \simeq a A_{1} B_{2} / \pi$, so one could in principle tune the harmonic $a$ by direct measurement of $h_{E}$ from EEHG-E.

\section{EXPERIMENT}

A layout of the experiment, carried out at the Next Linear Collider Test Accelerator (NLCTA) at SLAC $[13,15]$ is shown in Fig. 1. The electron beam with $\sim 20 \mathrm{pC}$ charge is generated in a 1.6 cell S-band $(2.856 \mathrm{GHz}$ rf frequency) photocathode gun with a UV laser (1 ps FWHM) and further accelerated to $120 \mathrm{MeV}$ on-crest $\left(\varphi_{\mathrm{rf}}=0\right)$ with $\mathrm{X}$-band $\left(\lambda_{\mathrm{rf}}=\mathrm{c} / 11.424 \mathrm{GHz}\right)$ linac structures. Velocity bunching after the gun compresses the beam to a measured rms length of $\sigma_{z} \simeq 160 \mathrm{fs}$. The beam has no linear rf chirp $\left(h_{1}=0\right)$ but extends over about $4^{\circ}$ of the X-band bucket and therefore has an rf-induced curvature characterized by $h_{2} \simeq 2.8 \times 10^{-5}$, for an assumed $\sigma_{E} \simeq 2 \mathrm{keV}$ slice energy spread [15]. At this level, the assumption that the curvature can be neglected in Eq. (2) is reasonable for $A_{1}>10$ according to the constraint that the full energy spread of the curved bunch be less than the induced energy modulation, $h_{2} \sigma_{z}^{2} k_{1}^{2}<A_{1} / \sqrt{6}$ (see the Appendix).

The EEHG portion of the beam line consists of two undulators ( $\mathrm{U} 1$ and $\mathrm{U} 2)$, three chicanes $(\mathrm{C} 1$ and $\mathrm{C} 2$ and $\mathrm{C} 3$ ), and one energy bend spectrometer. A Ti:sapphire laser (1 ps FWHM) with central wavelength at $800 \mathrm{~nm}$ is split into two pulses with a beam splitter. Chicane $\mathrm{C} 1$ is used to generate an orbit bump to allow injection of the first laser pulse $(\sim 100 \mu \mathrm{J})$ into the first undulator U1 (ten periods of $3.3 \mathrm{~cm}$ each and normalized magnetic field strength $K=1.82$ ), where the laser interacts with the electron beam to imprint a large sinusoidal energy modulation. Chicane C2 strongly overdisperses the modulated beam. The second, weaker laser pulse $(\ll 100 \mu \mathrm{J})$ then interacts with the e-beam in $\mathrm{U} 2$ at the third harmonic resonance (ten periods of $5.5 \mathrm{~cm}$ and $K=2.76$ ) to introduce a small energy modulation that flattens the energy bands, creating a projected energy distribution with fine grained structure [Figs. 1(F) and 1(H)]. The electron beam energy distribution is directly measured with a yttrium aluminum garnet (YAG) screen downstream of the energy spectrometer (1.5 $\mathrm{m}$ horizontal dispersion) which is $12 \mathrm{~m}$ downstream of $\mathrm{C} 2$.

Figure 2 shows the experimentally observed e-beam energy distribution in the case when the lasers are off [Fig. 2(a)], the first laser pulse is on [Fig. 2(b)], and when both lasers are on [Fig. 2(c)]. EEHG demands that the product $A_{1} B_{1}$ exceeds unity, and we choose $A_{1} \gg B_{1}$ in order to maximize our energy resolution and demonstrate the EEHG-E principle. Clear banding is observed in the energy distribution in Fig. 2(c) as a result of the modulation induced by the second laser, revealing energy structures even smaller than the unmodulated beam in Fig. 2(a). The measured energy spectra $\chi(\kappa)$ from the Fourier transform of the projected energy distributions are also shown. Excellent agreement is found between theory and the measurement, 

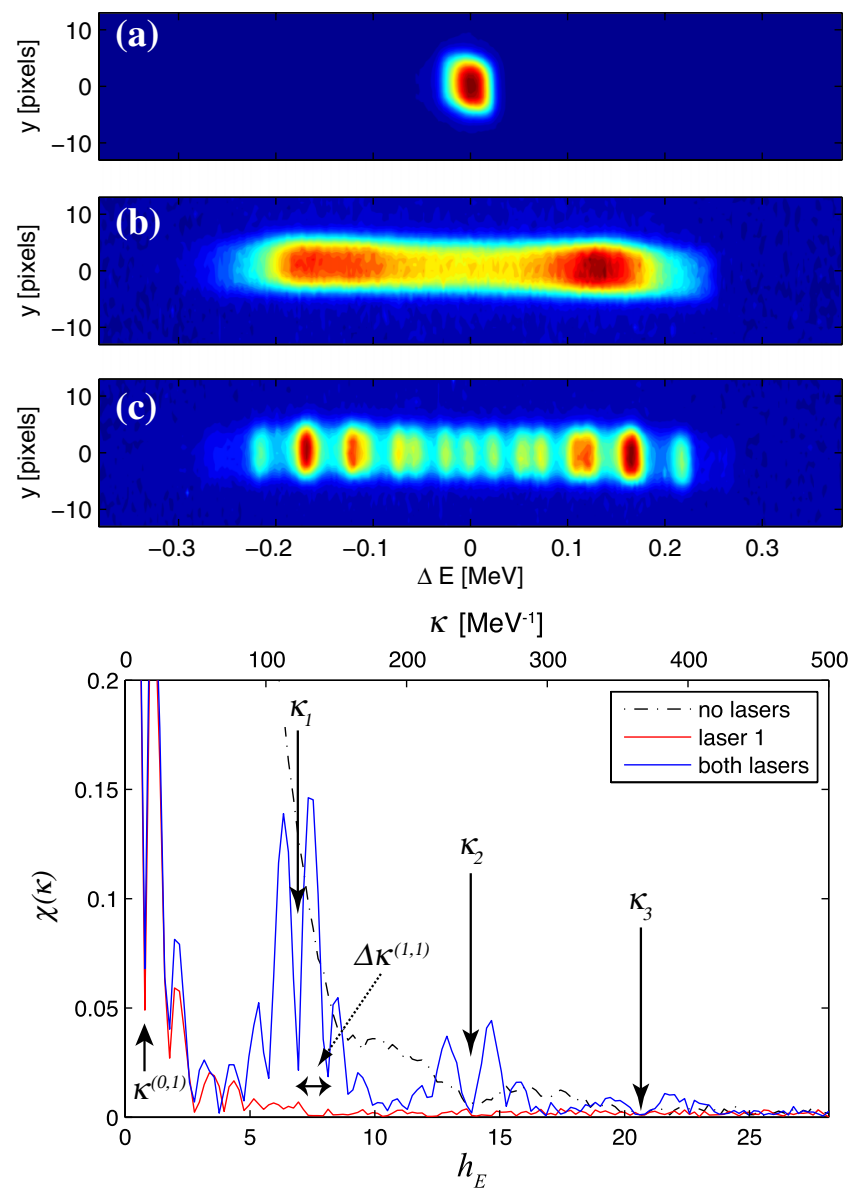

FIG. 2 (color online). Top: Measured e-beam energy distributions at bend spectrometer with (a) no laser modulation, (b) first laser on, and (c) both lasers on. Bottom: Energy spectra for each case, with the harmonic frequencies $\kappa_{n}=n k_{1} R_{56} / E_{0}$ identified from theory.

both in terms of the multipeaked spectrum, and in terms of the spectral zeros at $\kappa_{n}=n k_{1} R_{56} / E_{0}$ for each $n \neq 0$ predicted in Eq. (2). The dispersion of the chicane was $R_{56}=1.9 \mathrm{~mm}$, which corresponds to theoretically predicted spectral zeros at $\kappa_{n}=124.3, \quad 248.7$, and $373.0 \mathrm{MeV}^{-1}$. The experimentally measured values over 50 consecutive shots were $\kappa_{n}=124 \pm 1,249 \pm 2$, and $372 \pm 5 \mathrm{MeV}^{-1}$, respectively, or energy harmonics of $h_{E}=7,14$, and 21 . The experimental rms errors are close to the expected $6 \mathrm{keV}$ energy resolution at the screen, which should be less than $\delta E_{n} / 2$ to resolve nearby bands. We note that emittance cleaning of the beam with an aperture could be used in future experiments to reduce the horizontal beta function for better energy resolution, which was limited by contributions from the beam size and screen imaging system in our experiment.

The predicted dependence of the banding frequency on dispersion $\kappa_{n}=n k_{1} R_{56} / E_{0}$ was also tested by varying the $R_{56}$ of $\mathrm{C} 2$. An example of the observed energy distribution is shown in Fig. 3, as are the measured values of the $\kappa_{1}$ and $\kappa_{2}$ zeros for different dispersions that agree well with
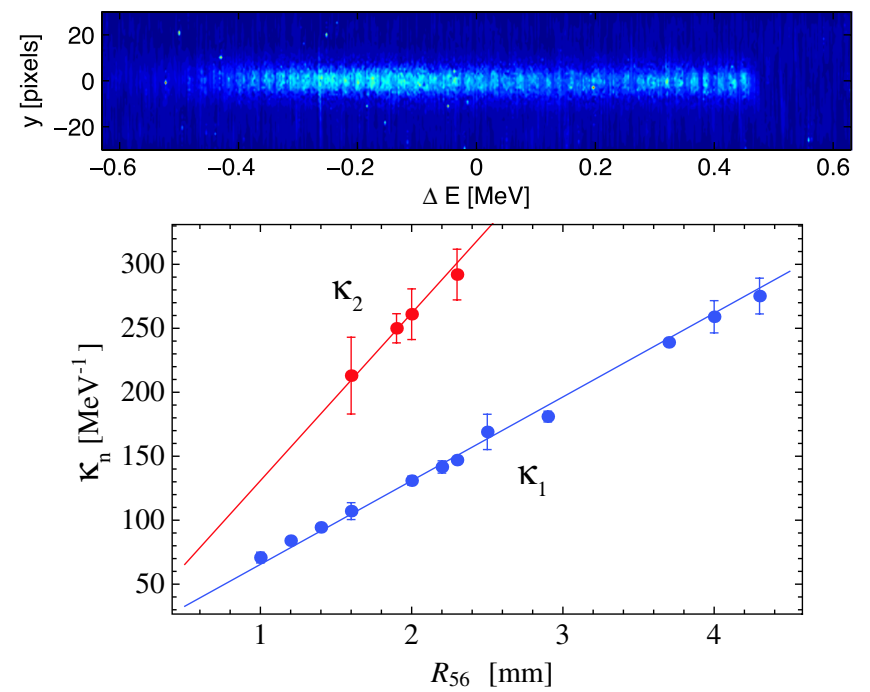

FIG. 3 (color online). Top: Observed e-beam distribution at the energy spectrometer for $R_{56}=4.3 \mathrm{~mm}$. Bottom: Measured energy banding frequency as a function of dispersion for the $n=1$ (blue) and $n=2$ (red) frequency zeros $\kappa_{n}$ in the banding spectrum. Points are from measurements with $3 \sigma$ error bars, and the lines are $\kappa_{n}=n k_{1} R_{56} / E_{0}$.

predictions. The data also show that $h_{E}=k_{1} R_{56} \Delta E_{1} / E_{0} \simeq$ 27 harmonics in the energy distribution are visible at $R_{56}=4.3 \mathrm{~mm}$ with $\Delta E_{1} \simeq 300 \mathrm{keV}$. With this highly sheared phase space, we note that a second modulation of comparable magnitude in $\mathrm{U} 2$ would therefore generate around $8 \%$ bunching at the $a \simeq h_{E} \pi / A_{1} B_{2}=80$ th harmonic $\left(\lambda_{1} / a \simeq 10 \mathrm{~nm}\right)$ with a dispersion in $\mathrm{C} 3$ of $R_{56}^{(2)} \simeq 55 \mu \mathrm{m}$.

The EEHG-E energy spectrum also provides a straightforward way to accurately quantify the laser induced energy modulations in order to calculate $h_{E}$ and optimize EEHG. Of course in principle, the amplitude of large individual laser modulations can be determined directly from the distance between the sharp double horns in the energy projection. However, if the modulation is small, or if the beam has either some global curvature from the rf (as it does in our experiment) or an rf chirp from $h_{1} \neq 0$, these horns become smeared and the precise modulation amplitude is obscured. An effective alternate solution is to look directly at the measured energy band spectrum, specifically the spectral zeros which are determined by the Bessel functions and are essentially independent of both $h_{1}$ and $h_{2}$. For example, $\Delta E_{1}$ from the first laser can be accurately found from the Bessel zeros $j_{n, s}$, which are the $s$ th zeros of $J_{n}$. From Eq. (2), these occur at the frequencies where $\left(\mu-n B_{1}\right) A_{1}=j_{n, s}$. Thus, we can define $\Delta \kappa^{(n, s)} \equiv \mid \kappa^{(n, s)}-$ $\kappa_{n} \mid=j_{n, s} / \Delta E_{1}$ as the frequency difference between the zeros $\kappa^{(n, s)}$ and the neighboring $\kappa_{n}$. This way, $\Delta E_{1}$ is determined simply by measuring $\Delta \kappa^{(n, s)}$ from the spectrum. First consider $A_{2}=0$, so that only the $n=0$ term in Eq. (2) matters. Then $\kappa_{0}=0$ and the first root is $j_{0,1}=2.4$, so the energy modulation amplitude in Fig. 2(b) is given simply by $\Delta E_{1}=2.4 / \kappa^{(0,1)}=177 \pm 14 \mathrm{keV}$, where 
$\kappa^{(0,1)} \simeq 13.5 \mathrm{MeV}^{-1}$ is the observed lowest frequency zero (Fig. 2 bottom, red line). This "root finding" procedure works with the second laser also on (Fig. 2 bottom, blue line), using the widths of the peaks near $\kappa_{n}$. From the first order peaks near $n=1$ in Fig. 2, for example, we measure a root separation of $\Delta \kappa^{(1,1)} \simeq 22 \mathrm{MeV}^{-1}$ which, with $j_{1,1}=$ 3.83 also gives $\Delta E_{1}=174 \pm 14 \mathrm{keV}$. Note that with $\Delta E_{1}=0$, the $j_{0, s}$ roots can also be used to measure the energy modulation of the second laser, which was found to be $\Delta E_{2} \simeq 20 \mathrm{keV}$, but was difficult to quantify exactly because it was about the same as the global energy spread.

To test the consistency between these amplitude measurements from the spectrum, we varied $\Delta E_{1}$ by adjusting the laser field in U1 with a linear polarizer, and calculated $\Delta E_{1}$ from several spectral zeros in the observed energy banding spectrum. Good mutual agreement was found. The positions of $\kappa_{1}$ and $\kappa_{2}$ were also confirmed to be independent of $\Delta E_{1}$, as expected from Eq. (2) and from EEHG theory, where the tuning of the harmonic number $a$ is insensitive to $A_{1}$ for $A_{1} B_{1} \gg 1$.

Finally, we note that the flattened multiband energy distribution created by this technique may have useful alternate applications. In Ref. [19], a multipeaked electron energy distribution with beamlets separated by more than the FEL bandwidth was used to generate a train of ultrashort pulses in the the FEL output. Similarly, ultrashort soft $\mathrm{x}$-ray pulses may also be amplified by a relativistic streaming instability driven by longitudinal space charge in a multiband energy structure [20]. An oscillatory energy distribution has also been shown to considerably reduce the FEL gain length, particularly for beams characterized by an otherwise large relative energy spread [21].
In summary, the energy banding spectrum appears to be a useful broadband tool to probe and characterize EEHG at an intermediate stage of the harmonic up-conversion process. We suggest that the EEHG-E technique can also be used to optimize and benchmark the subtle physics of EEHG while potentially also being used as an on-line diagnostic in tandem with the emission spectrum. While not accessible given the beam parameters of our experiment, undesirable effects like IBS and ISR may also be gauged for large e-beam currents and strong dispersions in future studies, provided that the energy resolution in the bend spectrometer is adequate to reveal the fine-scale structures. As an order of magnitude estimate, from the relation $h_{E}=2 \Delta E_{1} / \delta E \simeq a A_{1} B_{2} / \pi$, the minimum relative energy resolution required to measure banding structures that result in density bunching at the harmonic $a$ is $\delta E / 2 E_{0} \sim \lambda_{1} / 2 R_{56}$, where it is assumed that the harmonic is determined approximately by the ratio of the dispersions, $a \sim R_{56} / R_{56}^{(2)}$.

\section{ACKNOWLEDGMENTS}

We thank G. Stupakov for useful discussions. This work was supported by the U.S. DOE Office of Basic Energy Sciences using the NLCTA facility which is partly supported by U.S. DOE Office of High Energy Physics under Contract No. DE-AC02-76SF00515.

\section{APPENDIX}

The general solution of the energy banding spectrum in Eq. (1) is given by

$$
\chi(\mu)=\left|\sum_{n, m} \operatorname{Exp}\left[-\frac{\left(\mu+m K_{2} B_{1}\right)^{2}}{2}-\frac{\sigma_{z}^{2} k_{1}^{2}\left[\left(n+m K_{2}\right)-h_{1}\left(\mu+m K_{2} B_{1}\right)\right]^{2}}{2 \nu_{m}(\mu)}+i m \varphi\right] \frac{J_{n}\left(\mu A_{1}+m K_{2} B_{1} A_{1}\right)}{\sqrt{\nu_{m}(\mu)}} J_{m}\left(\mu A_{2}\right)\right|,
$$

where $K_{2}=k_{2} / k_{1}$ is the ratio of the two laser modulation frequencies and

$$
\nu_{m}(\mu)=1+2 i h_{2} k_{1}^{2} \sigma_{z}^{2}\left(\mu+m K_{2} B_{1}\right)
$$

quantifies the contribution from the initial rf curvature on the electron beam phase space. As with EEHG, the formal dependence on Bessel functions in Eq. (A1) is the inherent result of the sinusoidal structure of the laser modulations.

In the typical case where the electron bunch is long compared to the laser wavelength $\sigma_{z} k_{1} \gg 1$, only the terms for which $n=-m K_{2}$ contribute to the sum. Because both $n$ and $m$ are integers, this condition shows that the projected energy distribution reveals energy banding from the interplay of both lasers only when their wavelengths are integer multiples of each other. Otherwise the longitudinal projection of the fine-scale striations becomes washed out, and the energy banding structures are suppressed. In our experiment, both lasers originate from the same source and thus have the same wavelength $\left(K_{2}=1\right)$, so all the terms where $n=-m$ contribute to the spectrum.

In this case Eq. (A1) can be simplified:

$$
\chi(\mu)=\left|\sum_{n} \operatorname{Exp}\left[-\frac{\left(\mu-n B_{1}\right)^{2}}{2}\left(1+\frac{\sigma_{z}^{2} k_{1}^{2} h_{1}^{2}}{2 \nu_{-n}(\mu)}\right)\right] \frac{J_{n}\left(\mu A_{1}-n B_{1} A_{1}\right)}{\sqrt{\nu_{-n}(\mu)}} J_{-n}\left(\mu A_{2}\right)\right| .
$$


In the absence of rf-induced correlations $\left(h_{1}, h_{2}=0\right)$ then $\nu_{-n}(\mu)=1$ and this reduces to Eq. (2):

$\chi(\mu)=\left|\sum_{n} \operatorname{Exp}\left[-\frac{\left(\mu-n B_{1}\right)^{2}}{2}\right] J_{n}\left(\mu A_{1}-n B_{1} A_{1}\right) J_{-n}\left(\mu A_{2}\right)\right|$.

To obtain good contrast for diagnosing the energy bands, it is advantageous to make $A_{1}$ large since the first term $e^{-\xi^{2} / 2} J_{n}\left(\xi A_{1}\right)$ is increased for $A_{1} \geq 5$ (a similar behavior occurs in EEHG for the bunching factor). These peak values are localized near $\mu=n B_{1}$, therefore the optimal value of the second modulation is set by maximizing $J_{-n}\left(\mu A_{2}\right)$ near these frequencies. Because $J_{n}$ is maximized when the argument is $n+0.81 n^{1 / 3}$, the second laser modulation in EEHG-E should be $A_{2} \simeq(n+$ $\left.0.81 n^{1 / 3}\right) / n B_{1}$ to optimally reveal the finest-grained energy structures for diagnosing the EEHG process. Physically, this optimization has the effect of making the energy bands as flat as possible for a given $n$ to sharpen their projection within the sinusoidal modulation, and thus to enhance the signal for the higher harmonic $n>1$ terms. Assuming that a minimum relative energy resolution of $\lambda_{1} / 2 n R_{56}$ is maintained, one can then scan the dispersion $B_{2}$ to assess the effect of IBS on the energy bands, which depends primarily on the value of the final chicane strength $[16,17]$.

Finally, it is worth commenting on the contribution from the rf terms $h_{1}$ and $h_{2}$ on the energy structure. The linear term $h_{1}$ describes a bunch with a linear chirp prior to the first modulation, and behaves essentially like an additional energy spread that suppresses the banding structure when $h_{1} \sigma_{z} k_{1}$ becomes comparable to $A_{1} / \sqrt{2}$. A similar effect occurs for a quadratic bunch curvature when the global energy spread from curvature approaches the modulation amplitude, or when $\sqrt{3} h_{2} \sigma_{z}^{2} k_{1}^{2}$ is comparable to $A_{1} / \sqrt{2}$. Operating the rf accelerating structures on-crest avoids suppression from the $h_{1}$ term, while linearizing the phase space or operating with short bunches can avoid suppression from the $h_{2}$ term.

[1] G. Stupakov, Phys. Rev. Lett. 102, 074801 (2009).

[2] D. Xiang and G. Stupakov, Phys. Rev. ST Accel. Beams 12, 030702 (2009).

[3] L. H. Yu, Phys. Rev. A 44, 5178 (1991).

[4] E. Allaria, D. Castronovo, P. Cinquegrana, P. Craievich, M. Dal Forno, M. Danailov, G. D’Auria, A. Demidovich, G. De Ninno, S. Di Mitri et al., Nat. Photonics 7, 913 (2013).

[5] E. Allaria, G. D. Ninno, and D. Xiang, in Proceedings of the 31st International Free Electron Laser Conference
(FEL 09), Liverpool, UK (STFC Daresbury Laboratory, Warrington, 2009), pp. 39-42.

[6] Z. Zhao, in Proceedings of the 2nd International Particle Accelerator Conference, San Sebastián, Spain (EPS-AG, Spain, 2011), pp. 3011-3013.

[7] A. Zholents, B. Austin, K. Baptiste, J. Byrd, J. Corlett et al., in Proceedings of the 2nd International Particle Accelerator Conference, San Sebastián, Spain (Ref. [6]), pp. 775-777.

[8] R. Molo, M. Bakr, M. Höner, H. Huck, S. Khan, A. Nowaczyk, A. Schick, P. Ungelenk, and M. Zeinalzadeh, in Proceedings of the 33rd International Free Electron Laser Conference (FEL 11), SINAP, Shanghai, China, 2012, pp. 219-222.

[9] K. Hacker, S. Khan, H. Schlarb, P. V. der Meulen, P. Salen, A. Azima, G. A. Hamberg, and V. Ziemann, in Proceedings of the 33rd International Free Electron Laser Conference (FEL 11), SINAP, Shanghai, China, 2012, pp. 279-282.

[10] Q. Marksteiner, K. Bishofberger, B. Carlsten, L. Duffy, and N. Yampolsky, in Proceedings of the 33rd International Free Electron Laser Conference (FEL 11), SINAP, Shanghai, China, 2012, pp. 41-44.

[11] C. Evain, A. Loulergue, A. Nadji, J. Filhol, M. Couprie, and A. Zholents, New J. Phys. 14, 023003 (2012).

[12] E. Prat and S. Reiche, in Proceedings of the 33rd International Free Electron Laser Conference (FEL 11), SINAP, Shanghai, China, 2012, pp. 251-254.

[13] D. Xiang, E. Colby, M. Dunning, S. Gilevich, C. Hast, K. Jobe, D. McCormick, J. Nelson, T. O. Raubenheimer, K. Soong et al., Phys. Rev. Lett. 105, 114801 (2010).

[14] Z. T. Zhao, D. Wang, J. H. Chen, Z. H. Chen, H. X. Deng, J. G. Ding, C. Feng, Q. Gu, M. M. Huang, T. H. Lan et al., Nat. Photonics 6, 360 (2012).

[15] D. Xiang, E. Colby, M. Dunning, S. Gilevich, C. Hast, K. Jobe, D. McCormick, J. Nelson, T. O. Raubenheimer, K. Soong et al., Phys. Rev. Lett. 108, 024802 (2012).

[16] G. Stupakov, in Proceedings of the 33rd International Free Electron Laser Conference (FEL 11), SINAP, Shanghai, China, 2012, pp. 44-52.

[17] G. Dattoli and E. Sabia, Phys. Rev. ST Accel. Beams 16, 070702 (2013).

[18] The different local chirps generated by the laser also explain why a given harmonic frequency $a$ can be obtained with two different values of $B_{1}$ in EEHG.

[19] V. Petrillo, M. P. Anania, M. Artioli, A. Bacci, M. Bellaveglia, E. Chiadroni, A. Cianchi, F. Ciocci, G. Dattoli, D. Di Giovenale et al., Phys. Rev. Lett. 111, 114802 (2013).

[20] A. Marinelli, E. Hemsing, and J. B. Rosenzweig, Phys. Rev. Lett. 110, 064804 (2013).

[21] G. Stupakov, Y. Ding, and Z. Huang, in Proceedings of the 31st International Free Electron Laser Conference (FEL 09), Liverpool, UK (Ref. [5]), pp. 130-133. 